TRANSACTIONS OF THE

AMERICAN MATHEMATICAL SOCIETY

Volume 352, Number 5 , Pages 2145-2156

S 0002-9947(00)02306-0

Article electronically published on February 16, 2000

\title{
SOME PROPERTIES OF PARTITIONS IN TERMS OF CRANK
}

\author{
A. BÜLENT EKIN
}

\begin{abstract}
Let $N(r, m, n)$ (resp. $M(r, m, n))$ denote the number of partitions of $n$ whose ranks (resp. cranks) are congruent to $r$ modulo $m$. Atkin and Swinnerton-Dyer gave the relations between the numbers $N(r, m, m n+k)$ when $m=5,7$ and $0 \leq r, k<m$. Garvan gave the relations between the numbers $M(r, m, m n+k)$ when $m=5,7$, and $11,0 \leq r, k<m$. Here, we show that the methods of Atkin and Swinnerton-Dyer can be extended to prove the relations for the crank.
\end{abstract}

\section{INTRODUCTION}

Let $N(m, n)$ and $M(m, n)$ denote the number of partitions of $n$ with rank and crank $m$, respectively. We change this definition of $M(m, n)$ just a little, setting $M(0,1)=-1$ and $M(-1,1)=1=M(1,1)$, and modify $M(r, m, n)$ accordingly. We shall also suppose that the empty partition of 0 has rank 0.

For convenience, we write $N_{1}$ for $M$ and $N_{3}$ for $N$. So, by (12) of [9] and (1.11) of [2] when $k=1$, and by (2.12) of [3] when $k=3$, we have

$$
\sum_{m} \sum_{n \geq 0} N_{k}(m, n) z^{m} q^{n}=\left(\prod_{r=1}^{\infty} \frac{1}{1-q^{r}}\right)(1-z) \sum_{n}(-1)^{n} \frac{q^{n(k n+1) / 2}}{1-z q^{n}} .
$$

Here, and below, $\sum_{n}$ denotes a sum over all integers $n$, while $\sum_{n}^{\prime}$ denotes a sum over all non-zero integers $n$.

For odd positive integers $k$, defining $N_{k}(m, n)$ by (1.1), we observe that $N_{k}(m, n)$ $\geq 0$ for almost all $n$. If we put $z=1$ in (1.1), we find that

$$
\sum_{m} N_{k}(m, n)=p(n),
$$

where $p(n)$ is the number of partitions of $n$, and, replacing $z$ by $z^{-1}$ in (1.2),

$$
N_{k}(-m, n)=N_{k}(m, n) .
$$

Thus, one can ask whether there is a " $k$-rank" (1-rank $=$ crank, 3-rank $=$ rank) such that $N_{k}(m, n)$ counts the number of partitions of $n$ with $k$-rank $m$. This question has been answered by Garvan [8]

In [7], Garvan found a number of relations for the crank modulo 5, 7 and 11 analogous to those found by Dyson [5] for the rank modulo 5 and 7 . These relations were proved by Garvan using various results for theta functions, including

Received by the editors August 11, 1995 and, in revised form, January 6, 1998

2000 Mathematics Subject Classification. Primary 11P83.

(C)2000 American Mathematical Society 
Winquist's identity 11. Dyson's results were proved by Atkin and SwinnertonDyer [3] by relating certain mock-theta-like functions with elliptic-theta function identities. In this paper we show how Atkin and Swinnerton-Dyer's method may be extended to prove Garvan's crank relations. In the process we find some new relations for mock-theta-like functions.

Since we use the method of Atkin and Swinnerton-Dyer [3], we adopt their notations: $m$ will take the values 5,7 and 11, and the variables $q$ and $y$ are always related by the equation

$$
y=q^{m} \text {. }
$$

We shall regard any power series in $q$ as a polynomial of degree $m-1$ in $q$ whose coefficients are power series in $y$. Thus, any identity between two power series in $q$ can be regarded, on equating coefficients of powers of $q$, as equivalent to $m$ identities between power series in $y$. We write

$$
P(z, q):=\prod_{r=1}^{\infty}\left(1-z q^{r-1}\right)\left(1-z^{-1} q^{r}\right),
$$

so that $P(z, q)$ is a single-valued analytic function of $z$ in any ring-shaped region $0<z_{1} \leq|z| \leq z_{2}$, and satisfies

$$
P\left(z^{-1} q, q\right)=P(z, q), P(z q, q)=-z^{-1} P(z, q) .
$$

We also write

$$
\begin{aligned}
& P(a):=P_{m}(a):=P\left(y^{a}, y^{m}\right)=\prod_{r=1}^{\infty}\left(1-y^{m(r-1)+a}\right)\left(1-y^{m r-a}\right), \\
& P(0):=P_{m}(0):=\prod_{r=1}^{\infty}\left(1-y^{m r}\right)
\end{aligned}
$$

where $a$ is not a multiple of $m$. It should be noted that $P(0)$ is not the expression that would be obtained by writing 0 instead of $a$ in the definition of $P(a)$. From (1.5), we have

$$
P(m-a)=P(a), \quad P(-a)=P(m+a)=-y^{-a} P(a),
$$

which we shall use without explicit mention below.

\section{Preparation}

We define

$$
M(r):=\sum_{n \geq 0} M(r, m, n) q^{n}
$$

and

$$
S(a):=\sum_{n}^{\prime}(-1)^{n} \frac{q^{n(n+1) / 2+a n}}{1-q^{m n}} .
$$

The power series $S(a)$ differs only from (6.1) of [3] in that 3 is replaced by 1 , which appears in the power of $q$ in the numerator of (6.1) of [3]. As in [3], writing $-n$ for $n$ in (2.2), we find that

$$
S(a)=-S(m-1-a) .
$$


This gives

$$
S\left(\frac{m-1}{2}\right)=0 .
$$

Replacing $z$ by a primitive $m$-th root of unity in (1.1), we can see that

$$
\begin{aligned}
& M(0)=\mathbf{F}(S(0)-S(m-1)+1), \\
& M(r)=\mathbf{F}(S(r)-S(r-1)) \quad(r=1,2,3, \ldots, m-1),
\end{aligned}
$$

where $\mathbf{F}=\prod_{r=1}^{\infty}\left(1-q^{r}\right)^{-1}$ (see [10 for details). To express the power series $S(a)$ as a polynomial in $q$ of degree $m-1$ in $q$ whose coefficients are power series in $y$, we define (for any complex number $\zeta$ )

$$
T(z, \zeta, q):=\sum_{n}(-1)^{n} \frac{\zeta^{n} q^{n(n+1) / 2}}{1-z q^{n}},
$$

which is obviously an analytic function of $z$ in every region $0<r_{1} \leq|z| \leq r_{2}$ except for simple poles at point $z=q^{n}$. Also, let

$$
T^{*}(\zeta, q):=\sum_{n}^{\prime}(-1)^{n} \frac{\zeta^{n} q^{n(n+1) / 2}}{1-q^{n}} .
$$

Following the similar proof of (6.6) of [3] , we find

$$
\begin{aligned}
S\left(\frac{m-1}{2}-b\right)= & (-1)^{b} q^{b(m-b) / 2} T\left(q^{m b}, 1, q^{m^{2}}\right) \\
& +T^{*}\left(q^{-m b}, q^{m^{2}}\right)+q^{m b} T\left(q^{2 m b}, q^{m b}, q^{m^{2}}\right) \\
& +\sum_{\substack{a=1 \\
a \neq \mp b \bmod (m)}}^{(m-1) / 2}(-1)^{a+b} q^{(a-b)(a+b-m) / 2}\left\{q^{m a} T\left(q^{m(b+a)}, q^{m a}, q^{m^{2}}\right)\right. \\
& \left.+T\left(q^{m(b-a)}, q^{-m a}, q^{m^{2}}\right)\right\},
\end{aligned}
$$

It follows from Lemma 2 of $[9$ that

$$
\zeta T(z \zeta, \zeta, q)+T\left(z \zeta^{-1}, \zeta^{-1}, q\right)=\frac{P(z ; q) P\left(\zeta^{2} ; q\right) \mathbf{F}^{-2}}{P\left(z \zeta^{-1} ; q\right) P(z \zeta ; q) P(\zeta ; q)},
$$

and that

$$
T(z, 1, q)=\frac{\mathbf{F}^{-2}}{P(z ; q)} .
$$

We now write

$$
h(z ; q):=T^{*}\left(z^{-1}, q\right)+z T\left(z^{2}, z, q\right) .
$$

\section{Lemma 1.}

$$
\begin{aligned}
h(z ; q)-h(z q ; q) & =1 \\
h(z ; q)+h\left(z^{-1} ; q\right) & =0 \\
h(z ; q)+h\left(z^{-1} q ; q\right) & =-1 \\
3 h(z ; q)-h\left(z^{3} ; q\right) & =\frac{P^{3}\left(z^{2} ; q\right) \mathbf{F}^{-2}}{P^{3}(z ; q) P\left(z^{3} ; q\right)}-\frac{P^{3}\left(z^{4} ; q\right) \mathbf{F}^{-2}}{P^{3}\left(z^{2} ; q\right) P\left(z^{6} ; q\right)} .
\end{aligned}
$$


Proof. (i). By Jacobi's triple product identity (Thm.2.8 in) [1] we have

$$
-P\left(z^{-1} ; q\right) \mathbf{F}^{-1}=-\sum_{n}^{\prime}(-1)^{n} \frac{z^{-n} q^{n(n-1) / 2}}{1-q^{n}}+\sum_{n}^{\prime}(-1)^{n} \frac{z^{-n} q^{n(n+1) / 2}}{1-q^{n}}-1
$$

and

$$
\begin{aligned}
P\left(z^{-1} ; q\right) \mathbf{F}^{-1}= & \sum_{n}(-1)^{n} \frac{z^{n} q^{n(n+1) / 2}}{1-z^{2} q^{n+1}}\left(1-z^{2} q^{n+1}\right) \\
= & \sum_{n}(-1)^{n} \frac{z^{n} q^{n(n+1) / 2}}{1-z^{2} q^{n+1}}-\sum_{n}(-1)^{n} \frac{z^{n+2} q^{(n+1)(n+2) / 2}}{1-z^{2} q^{n+1}} \\
& (\text { replace } n \text { by } n+1) \quad(\text { replace } n \text { by } n-1) \\
= & -z q \sum_{n}(-1)^{n} \frac{(z q)^{n} q^{n(n+1) / 2}}{1-z^{2} q^{n+2}}+z \sum_{n}(-1)^{n} \frac{z^{n} q^{n(n+1) / 2}}{1-z^{2} q^{n}} .
\end{aligned}
$$

If we add (2.12) and (2.13), we have $(i)$. (ii) is trivial and (iii) is consequence of (i) and $(i i)$.

For $(i v)$, let $f_{L}(z)$ and $f_{R}(z)$ denote the left and right sides of $(i v)$. Then $(i)$ shows that $f_{L}(z q)=f_{L}(z)$. By (1.5), $f_{R}(z q)=f_{R}(z)$. Now, $f_{L}(z)-f_{R}(z)$ is free from poles and so, by Lemma 2 of [3], either is free from zeros or is identically zero. By using (1.5), we find that $f_{R}\left(z^{-1}\right)=-f_{R}(z)$, and by (ii) $h\left(z^{-1} ; q\right)=-h(z ; q)$. Therefore $z=1$ is a zero of $f_{L}(z)-f_{R}(z)$.

Consequently, with the help of (2.9)-(2.11) with $z=q^{m a}, \zeta=q^{m b}$, and $q^{m^{2}}$ for $q$, we have

$$
\begin{aligned}
S\left(\frac{m-1}{2}-b\right)= & h\left(q^{m b} ; q^{m^{2}}\right)+(-1)^{b} q^{b(m-b) / 2} \frac{P_{m}^{2}(0)}{P_{m}(b)} \\
& +\sum_{\substack{a=1 \\
a \neq \neq b \bmod (m)}}^{(m-1) / 2}(-1)^{a+b} q^{(a-b)(a+b-m) / 2} \frac{P_{m}(b) P_{m}(2 a) P_{m}^{2}(0)}{P_{m}(b-a) P_{m}(b+a) P_{m}(a)} .
\end{aligned}
$$

We shall need the following, which is Lemma 6 in [3]:

$$
\mathbf{F}^{-1}=(-1)^{\lambda} q^{\lambda(3 \lambda+\mu) / 2} P_{m}(0)\left[1+\sum_{c=1}^{(m-1) / 2}(-1)^{c} q^{c(3 c-m) / 2} \frac{P_{m}(2 c)}{P_{m}(c)}\right]
$$

where $m=6 \lambda+\mu, \lambda$ is a positive integer and $\mu=\mp 1$.

We are now in a position to state and prove the results for crank of ordinary partition in the cases of modulo 5, 7 and 11. For convenience, we shall write

$$
R_{i j}(k):=\sum_{n \geq 0}(M(i, m, m n+k)-M(j, m, m n+k)) y^{n},
$$

and also

$$
h(a):=h\left(y^{a} ; y^{m}\right)
$$


so that Lemma 1, with $q$ replaced by $y^{m}$ and $z$ by $y^{a}$, states that

$$
\begin{aligned}
h(a)-h(m+a) & =1 \\
h(a)+h(m-a) & =-1 \\
3 h(a)-h(3 a) & =\frac{P^{3}(2 a) P^{2}(0)}{P^{3}(a) P(3 a)}-\frac{P^{3}(4 a) P^{2}(0)}{P^{3}(2 a) P(6 a)} .
\end{aligned}
$$

\section{SOME RESUlTS FOR CRANKS MODUlO 5}

Taking $m=5$ in (2.5), with the help of (2.3) and (2.4), we find that

$$
\begin{aligned}
& M(0)=\mathbf{F}(2 S(0)+1), \\
& M(1)=\mathbf{F}(S(1)-S(0)), \\
& M(2)=-\mathbf{F}(S(1)) .
\end{aligned}
$$

Taking $m=5$ and $b=2,1$ in (2.14), we have

$$
\begin{aligned}
& S(0)=h(2)-q \frac{P^{2}(0) P(2)}{P^{2}(1)}+q^{3} \frac{P^{2}(0)}{P(2)}, \\
& S(1)=h(1)-q^{2} \frac{P^{2}(0)}{P(1)}+q^{4} \frac{P^{2}(0) P(1)}{P^{2}(2)} .
\end{aligned}
$$

Putting $a=1$ in (2.18) and (2.20) with $m=5$, and $a=2$ in (2.19) and (2.20) with $m=5$, we also obtain

$$
\begin{aligned}
& h(1)=-\frac{1}{5}+\frac{1}{5}\left\{\frac{P^{2}(0) P^{2}(2)}{P^{3}(1)}+2 y \frac{P^{2}(0) P^{2}(1)}{P^{3}(2)}\right\}, \\
& h(2)=-\frac{2}{5}+\frac{1}{5}\left\{2 \frac{P^{2}(0) P^{2}(2)}{P^{3}(1)}-y \frac{P^{2}(0) P^{2}(1)}{P^{3}(2)}\right\} .
\end{aligned}
$$

After all these preparations the following is easily proven.

\section{Theorem 1.}

$$
\begin{aligned}
& R_{01}(0)=\frac{P(2) P(0)}{P^{2}(1)} \\
& R_{01}(1)=-2 \frac{P(0)}{P(1)} \\
& R_{12}(1)=\frac{P(0)}{P(1)} \\
& R_{12}(2)=-\frac{P(0)}{P(2)} \\
& R_{01}(3)=-R_{12}(3)=\frac{P(1) P(0)}{P^{2}(2)}
\end{aligned}
$$

and all other functions $R_{b, b+1}(d)$, where $b=0$ or 1 , are zero.

By (3.1), to prove the theorem we only have to show that

$$
\begin{aligned}
& 3 S(0)-S(1)+1=\left\{\frac{P(0) P(2)}{P^{2}(1)}-2 q \frac{P(0)}{P(1)}+q^{3} \frac{P(0) P(1)}{P^{2}(2)}\right\} \mathbf{F}^{-1}, \\
& 2 S(1)-S(0)=\left\{q \frac{P(0)}{P(1)}-q^{2} \frac{P(0)}{P(2)}-q^{3} \frac{P(0) P(1)}{P^{2}(2)}\right\} \mathbf{F}^{-1} .
\end{aligned}
$$


Since by (2.15) we have

$$
\mathbf{F}^{-1}=P(0)\left\{\frac{P(2)}{P(1)}-q-q^{2} \frac{P(1)}{P(2)}\right\},
$$

these are respectively equivalent to

$$
\begin{aligned}
& 3 h(2)-h(1)+1=\frac{P^{2}(0) P^{2}(2)}{P^{3}(1)}-y \frac{P^{2}(0) P^{2}(1)}{P^{3}(2)}, \\
& 2 h(1)-h(2)=y \frac{P^{2}(0) P^{2}(1)}{P^{3}(2)},
\end{aligned}
$$

which are true by (3.3). This proves the theorem.

\section{Some ReSUlts FOR CRANKS MOdUlo 7}

Here and in the next section we need the following for simplifications, which is Lemma 4 of [3],

$$
\begin{aligned}
P^{2}(b) P(c+d) P(c-d) & -P^{2}(c) P(b+d) P(b-d) \\
& +y^{c-d} P^{2}(d) P(b+c) P(b-c)=0,
\end{aligned}
$$

where none of $b, c, d, b \mp c, c \mp d, b \mp d$ is divisible by $m$. This gives, for $(b, c, d)=(3,2,1)$,

$$
P(1) P^{3}(3)-P(3) P^{3}(2)+y P(2) P^{3}(1)=0 .
$$

As in the previous section, taking $m=7$ in (2.5), with the help of (2.3) and (2.4) we find that

$$
\begin{aligned}
& M(0)=\mathbf{F}(2 S(0)+1), \\
& M(1)=\mathbf{F}(S(1)-S(0)), \\
& M(2)=\mathbf{F}(S(2)-S(1)), \\
& M(3)=-\mathbf{F}(S(2)) .
\end{aligned}
$$

Taking $m=7$ and $b=3,2$, and 1 in (2.14), we have

$$
\begin{aligned}
& S(0)=h(3)-q \frac{P^{2}(0) P^{2}(3)}{P^{2}(2) P(1)}+q^{3} \frac{P^{2}(0)}{P(1)}-q^{6} \frac{P^{2}(0)}{P(3)}, \\
& S(1)=h(2)-q^{2} \frac{P^{2}(0) P^{2}(2)}{P^{2}(1) P(3)}+q^{5} \frac{P^{2}(0)}{P(2)}+q^{6} \frac{P^{2}(0)}{P(3)}, \\
& S(2)=h(1)-q^{3} \frac{P^{2}(0)}{P(1)}+q^{5} \frac{P^{2}(0)}{P(2)}-q^{11} \frac{P^{2}(0) P^{2}(1)}{P^{2}(3) P(2)} .
\end{aligned}
$$

Putting $a=1,2$ and 3 , respectively, in (2.18)-(2.20) with $m=7$, and using (4.2), we obtain

$$
\begin{aligned}
& h(1)=-\frac{1}{7}+\frac{1}{7} P^{2}(0)\left\{\frac{P(3)}{P^{2}(1)}+3 y \frac{P(1)}{P^{2}(2)}+2 y \frac{P(2)}{P^{2}(3)}\right\}, \\
& h(2)=-\frac{2}{7}+\frac{1}{7} P^{2}(0)\left\{2 \frac{P(3)}{P^{2}(1)}-y \frac{P(1)}{P^{2}(2)}-3 y \frac{P(2)}{P^{2}(3)}\right\}, \\
& h(3)=-\frac{3}{7}+\frac{1}{7} P^{2}(0)\left\{3 \frac{P(3)}{P^{2}(1)}+2 y \frac{P(1)}{P^{2}(2)}-y \frac{P(2)}{P^{2}(3)}\right\} .
\end{aligned}
$$


After all these preparations the following is easily proven.

\section{Theorem 2.}

$$
\begin{aligned}
& R_{01}(0)=\frac{P(3) P(0)}{P(1) P(2)} \\
& R_{01}(1)=-2 \frac{P(0)}{P(1)} \\
& R_{12}(1)=\frac{P(0)}{P(1)} \\
& R_{12}(2)=-R_{23}(2)=-\frac{P(2) P(0)}{P(1) P(3)} \\
& R_{01}(3)=-R_{23}(3)=\frac{P(0)}{P(2)} \\
& R_{01}(4)=-R_{12}(4)=\frac{P(0)}{P(3)} \\
& R_{01}(6)=-R_{12}(6)=R_{23}(6)=-\frac{P(1) P(0)}{P(2) P(3)}
\end{aligned}
$$

and all other functions $R_{b, b+1}(d)$, where $0 \leq b \leq 2$, are zero.

To prove the theorem we only consider the three pairs of values $(i, j)=(0,1)$, $(1,2)$ and $(2,3)$ in (2.16). So, by (4.3), we only have to show that

$$
3 S(0)-S(1)+1=\left\{\frac{P(0) P(3)}{P(1) P(2)}-2 q \frac{P(0)}{P(1)}+q^{3} \frac{P(0)}{P(2)}+q^{4} \frac{P(0)}{P(3)}-q^{6} \frac{P(0) P(1)}{P(2) P(3)}\right\} \mathbf{F}^{-1},
$$

$$
\begin{aligned}
& 2 S(1)-S(2)-S(0)=\left\{q \frac{P(0)}{P(1)}-q^{2} \frac{P(0) P(2)}{P(1) P(3)}-q^{4} \frac{P(0)}{P(3)}+q^{6} \frac{P(0) P(1)}{P(2) P(3)}\right\} \mathbf{F}^{-1}, \\
& 2 S(2)-S(1)=\left\{q^{2} \frac{P(0) P(2)}{P(1) P(3)}-q^{3} \frac{P(0)}{P(2)}-q^{6} \frac{P(0) P(1)}{P(2) P(3)}\right\} \mathbf{F}^{-1}
\end{aligned}
$$

Now by (2.15) we have

$$
\mathbf{F}^{-1}=P(0)\left\{\frac{P(2)}{P(1)}-q \frac{P(3)}{P(2)}-q^{2}+q^{5} \frac{P(1)}{P(3)}\right\} .
$$

Substituting this in each of (4.13)-4.15) and equating coefficients of powers of $q$, we have 21 equations to prove. The coefficients of $q^{0}$ give us respectively

$$
\begin{aligned}
3 h(3)-h(2)+1 & =P^{2}(0)\left\{y \frac{P(1)}{P^{2}(2)}+\frac{P(3)}{P^{2}(1)}\right\}, \\
2 h(2)-h(1)-h(3) & =-P^{2}(0) y\left\{\frac{P(2)}{P^{2}(3)}+\frac{P(1)}{P^{2}(2)}\right\}, \\
2 h(1)-h(2) & =-P^{2}(0) y\left\{\frac{P(2)}{P^{2}(3)}+\frac{P(1)}{P^{2}(2)}\right\},
\end{aligned}
$$


which are true by (4.5). All the other equations are trivially satisfied except for the coefficients of $q, q^{2}$ and $q^{4}$ in (4.13), of $q$ and $q^{2}$ in (4.14), and of $q^{4}$ in (4.15). The coefficients of $q, q^{2}$ and $q^{4}$ are respectively

$$
\begin{aligned}
& P^{2}(0)\left\{\frac{P(2)}{P^{2}(1)}-\frac{P^{2}(3)}{P(1) P^{2}(2)}-y \frac{P(1)}{P(2) P(3)}\right\}=0, \\
& P^{2}(0)\left\{y \frac{P(1)}{P^{2}(3)}-\frac{P^{2}(2)}{P(3) P^{2}(1)}+\frac{P(3)}{P(1) P(2)}\right\}=0, \\
& P^{2}(0)\left\{\frac{P(3)}{P^{2}(2)}+y \frac{P^{2}(1)}{P(2) P^{2}(3)}-\frac{P(2)}{P(1) P(3)}\right\}=0,
\end{aligned}
$$

and each of them reduces to (4.2). This proves the theorem.

\section{SOME RESUlts FOR CRANKS MOdUlo 11}

As in the previous section, taking $m=11$ in (2.5), with the help of (2.3) and (2.4) we find that

$$
\begin{aligned}
& M(0)=\mathbf{F}(2 S(0)+1), \\
& M(1)=\mathbf{F}(S(1)-S(0)), \\
& M(2)=\mathbf{F}(S(2)-S(1)), \\
& M(3)=\mathbf{F}(S(3)-S(2)), \\
& M(4)=\mathbf{F}(S(4)-S(3)), \\
& M(5)=-\mathbf{F}(S(4)) .
\end{aligned}
$$

Taking $m=11$ and $b=5,4,3,2$ and 1 respectively in (2.14), we have

$$
\begin{aligned}
S(0)=h(5) & -q \frac{P^{2}(0) P(3) P(5)}{P(1) P(2) P(4)}+q^{3} \frac{P^{2}(0) P^{2}(5)}{P(2) P^{2}(3)}-q^{4} y \frac{P^{2}(0)}{P(5)} \\
& -q^{6} \frac{P^{2}(0) P(5)}{P(2) P(3)}+q^{10} \frac{P^{2}(0) P(2)}{P(1) P(4)} \\
S(1)=h(4) & -q^{2} \frac{P^{2}(0) P(5)}{P(1) P(3)}+q^{3} y \frac{P^{2}(0)}{P(4)}+q^{5} \frac{P^{2}(0) P^{2}(4)}{P(5) P^{2}(2)} \\
& -q^{9} \frac{P^{2}(0) P(2) P(4)}{P(1) P(3) P(5)}+q^{10} \frac{P^{2}(0) P(4)}{P(2) P(5)}, \\
S(2)=h(3) & -q y \frac{P^{2}(0)}{P(3)}-q^{3} \frac{P^{2}(0) P(3) P(4)}{P(1) P(2) P(5)}+q^{7} \frac{P^{2}(0) P(3)}{P(1) P(4)} \\
& -q^{8} y \frac{P^{2}(0) P(1)}{P(2) P(5)}+q^{0} \frac{P^{2}(0) P^{2}(3)}{P(1) P^{2}(4)}, \\
S(3)=h(2) & +q^{4} \frac{P^{2}(0) P^{2}(2)}{P(3) P^{2}(1)}+q^{5} y^{2} \frac{P^{2}(0) P(1) P(2)}{P(3) P(4) P(5)}-q^{6} y \frac{P^{2}(0) P(3)}{P(4) P(5)} \\
& +q^{8} \frac{P^{2}(0) P(2)}{P(1) P(3)}+q^{9} \frac{P^{2}(0)}{P(2)}, \\
S(4)=h(1) & -q y^{3} \frac{P^{2}(0) P^{2}(1)}{P(4) P^{2}(5)}+q^{2} y^{2} \frac{P^{2}(0) P(1)}{P(4) P(5)}-q^{4} y \frac{P^{2}(0) P(1) P(5)}{P(2) P(3) P(4)} \\
& -q^{5} \frac{P^{2}(0)}{P(1)}+q^{7} \frac{P^{2}(0) P(4)}{P(2) P(3)} .
\end{aligned}
$$




\section{Theorem 3.}

$$
\begin{gathered}
R_{01}(0)=\frac{P(0)}{P(1)} \\
-\frac{1}{2} R_{01}(1)=R_{12}(1)=\frac{P(5) P(0)}{P(2) P(3)} \\
R_{12}(2)=-R_{23}(2)=-\frac{P(3) P(0)}{P(1) P(4)} \\
R_{01}(3)=-R_{23}(3)=R_{34}(3)=\frac{P(2) P(0)}{P(1) P(3)} \\
R_{01}(4)=-R_{12}(4)=R_{23}(4)=-R_{34}(4)=R_{45}(4)=\frac{P(0)}{P(2)}, \\
R_{12}(5)=-R_{23}(5)=R_{34}(5)=-R_{45}(5)=\frac{P(4) P(0)}{P(2) P(5)}, \\
R_{01}(7)=-R_{12}(7)=R_{34}(7)=-R_{45}(7)=-\frac{P(0)}{P(3)}, \\
R_{01}(8)=-R_{12}(8)=R_{23}(8)=-R_{45}(8)=-y \frac{P(1) P(0)}{P(4) P(5)} \\
R_{01}(9)=-R_{34}(9)=R_{45}(9)=-\frac{P(0)}{P(4)} \\
R_{23}(10)=-R_{34}(10)=\frac{P(0)}{P(5)}
\end{gathered}
$$

and all other functions $R_{b, b+1}(d)$, where $0 \leq b \leq 4$, are zero.

Since $R_{i j}(k)=-R_{j i}(k)$ and $R_{i s}(k)+R_{s j}(k)=R_{i j}(k)$, to prove the theorem it is sufficient to consider the five pairs of values $(i, j)=(0,5),(1,5),(2,5),(3,5)$ and $(4,5)$, so we have to prove

$$
\begin{aligned}
2 S(0) & +S(4)+1 \\
= & \left\{\frac{P(0)}{P(1)}-q \frac{P(0) P(5)}{P(2) P(3)}+q^{3} \frac{P(0) P(2)}{P(1) P(3)}+q^{4} \frac{P(0)}{P(2)}-q^{9} \frac{P(0)}{P(4)}\right\} \mathbf{F}^{-1},
\end{aligned}
$$

$$
S(1)+S(4)-S(0)=\left\{q \frac{P(0) P(5)}{P(2) P(3)}+q^{7} \frac{P(0)}{P(3)}+q^{8} y \frac{P(0) P(1)}{P(4) P(5)}\right\} \mathbf{F}^{-1},
$$

$$
S(2)+S(4)-S(1)=\left\{q^{2} \frac{P(0) P(3)}{P(1) P(4)}+q^{4} \frac{P(0)}{P(2)}-q^{5} \frac{P(0) P(4)}{P(2) P(5)}\right\} \mathbf{F}^{-1},
$$

$$
S(3)+S(4)-S(2)=\left\{q^{3} \frac{P(0) P(2)}{P(1) P(3)}+q^{8} y \frac{P(0) P(1)}{P(4) P(5)}-q^{10} y \frac{P(0)}{P(5)}\right\} \mathbf{F}^{-1},
$$

$$
\begin{aligned}
2 S(4) & -S(3) \\
= & \left\{q^{4} \frac{P(0)}{P(2)}-q^{5} \frac{P(0) P(4)}{P(2) P(5)}+q^{7} \frac{P(0)}{P(3)}+q^{8} y \frac{P(0) P(1)}{P(4) P(5)}-q^{9} \frac{P(0)}{P(4)}\right\} \mathbf{F}^{-1} .
\end{aligned}
$$


By (2.15) with $m=11(\lambda=2$ and $\mu=-1)$, we have

$$
\mathbf{F}^{-1}=P(0)\left\{\frac{P(4)}{P(2)}-q \frac{P(2)}{P(1)}-q^{2} \frac{P(5)}{P(3)}-q^{4} y \frac{P(1)}{P(5)}+q^{5}+q^{7} \frac{P(3)}{P(4)}\right\} .
$$

Substituting (5.18) in each of (5.13)-(5.17) and equating the coefficients of powers of $q$, we have 55 equations to prove. To do this we need the following ten identities, which can be found by taking $(b, c, d)=(5,4,1), .(5,4,2),(4,3,1),(5,3,2),(3,2,1)$, $(5,3,1),(5,4,3),(5,2,1),(4,3,2)$ and $(4,2,1)$ in (4.1):

$$
\begin{gathered}
P(3) P^{3}(5)-P(5) P^{3}(4)+y^{3} P(2) P^{3}(1)=0, \\
P(2) P^{3}(5)-P(3) P^{3}(4)+y^{2} P(1) P^{3}(2)=0, \\
P(2) P^{3}(4)-P(5) P^{3}(3)+y^{2} P(4) P^{3}(1)=0, \\
P(1) P^{3}(5)-P(4) P^{3}(3)+y P(3) P^{3}(2)=0, \\
P(1) P^{3}(3)-P(4) P^{3}(2)+y P(5) P^{3}(1)=0 . \\
P(2) P(4) P^{2}(5)-P(4) P(5) P^{2}(3)+y^{2} P(2) P(3) P^{2}(1)=0, \\
P(1) P(4) P^{2}(5)-P(2) P(3) P^{2}(4)+y P(1) P(2) P^{2}(3)=0, \\
P(1) P(3) P^{2}(5)-P(4) P(5) P^{2}(2)+y P(3) P(4) P^{2}(1)=0, \\
P(1) P(5) P^{2}(4)-P(2) P(5) P^{2}(3)+y P(1) P(4) P^{2}(2)=0, \\
P(1) P(3) P^{2}(4)-P(3) P(5) P^{2}(2)+y P(2) P(5) P^{2}(1)=0 .
\end{gathered}
$$

Putting $a=1,2,3,4$ and 5, respectively, in (2.18)-2.20) with $m=11$, and using (4.2), we obtain

$$
\begin{aligned}
& 3 h(1)-h(3)=B_{1}, \\
& 3 h(2)+h(5)=B_{2}-1, \\
& 3 h(3)+h(2)=B_{3}-1, \\
& 3 h(4)-h(1)=B_{4}-1, \\
& 3 h(5)-h(4)=B_{5}-1,
\end{aligned}
$$

where

$$
B_{i}=\frac{P^{3}(2 i) P^{2}(0)}{P^{3}(i) P(3 i)}-\frac{P^{3}(4 i) P^{2}(0)}{P^{3}(2 i) P(6 i)} \quad(i=1,2, \ldots, 5) .
$$

The solution of (5.21) is

$$
\begin{aligned}
h(1) & =-\frac{1}{11}+\frac{1}{242}\left(81 B_{1}-9 B_{2}+27 B_{3}+B_{4}+3 B_{5}\right), \\
h(2) & =-\frac{2}{11}+\frac{1}{242}\left(-3 B_{1}+81 B_{2}-B_{3}-9 B_{4}-27 B_{5}\right), \\
h(3) & =-\frac{3}{11}+\frac{1}{242}\left(B_{1}-27 B_{2}+81 B_{3}+3 B_{4}+9 B_{5}\right), \\
h(4) & =-\frac{4}{11}+\frac{1}{242}\left(27 B_{1}-3 B_{2}+9 B_{3}+81 B_{4}+B_{5}\right), \\
h(5) & =-\frac{5}{11}+\frac{1}{242}\left(9 B_{1}-B_{2}+3 B_{3}+27 B_{4}+81 B_{5}\right) .
\end{aligned}
$$


We simplify (5.23) by using some results of 4 as follows: Write

$$
\begin{gathered}
r=-y^{2} \frac{P(1)}{P(3) P(5)}, \quad s=-y \frac{P(2)}{P(1) P(5)}, \quad t=\frac{P(4)}{P(1) P(2)}, \\
u=y \frac{P(3)}{P(2) P(4)}, \quad v=y \frac{P(5)}{P(3) P(4)} .
\end{gathered}
$$

Now, dividing (b1)-(b5) respectively by

$$
\begin{gathered}
y^{-1} P(2) P(3) P(5) P^{2}(4), \quad P(1) P(3) P(4) P^{2}(2), \quad y^{-1} P(1) P(4) P(5) P^{2}(3), \\
P(2) P(4) P(5) P^{2}(1), \quad y^{-1} P(1) P(2) P(3) P^{2}(5)
\end{gathered}
$$

respectively, and dividing $(a 1)-(a 5)$ by

$$
\begin{gathered}
y^{-1} P(2) P(4) P^{3}(5), \quad P(3) P(5) P^{3}(2), \quad y^{-1} P(1) P(5) P^{3}(4), \\
P(1) P(2) P^{3}(3), \quad P(3) P(4) P^{3}(1)
\end{gathered}
$$

respectively, we find that

$$
\begin{aligned}
& B_{1}=(r+u+v-s) P^{2}(0), \\
& B_{2}=(t-r-s-v) P^{2}(0), \\
& B_{3}=(t+u+s-v) P^{2}(0), \\
& B_{4}=(t+r+s-u) P^{2}(0), \\
& B_{5}=(u+v+t-r) P^{2}(0) .
\end{aligned}
$$

Thus, (5.23) becomes

$$
\begin{aligned}
& h(1)=-\frac{1}{11}+\frac{1}{11}(4 r-2 s+t+5 u+3 v) P^{2}(0), \\
& h(2)=-\frac{2}{11}+\frac{1}{11}(-3 r-4 s+2 t-u-5 v) P^{2}(0), \\
& h(3)=-\frac{3}{11}+\frac{1}{11}(r+5 s+3 t+4 u-2 v) P^{2}(0), \\
& h(4)=-\frac{4}{11}+\frac{1}{11}(5 r+3 s+4 t-2 u+v) P^{2}(0), \\
& h(5)=-\frac{5}{11}+\frac{1}{11}(-2 r+s+5 t+3 u+4 v) P^{2}(0) .
\end{aligned}
$$

Therefore,

$$
\begin{aligned}
2 h(5)+h(1)+1 & =\left\{\frac{P(4)}{P(2) P(1)}+y \frac{P(3)}{P(2) P(4)}+y \frac{P(5)}{P(3) P(4)}\right\} P^{2}(0), \\
h(4)+h(1)-h(5) & =y^{2} \frac{P(1) P^{2}(0)}{P(3) P(5)}, \\
h(3)+h(1)-h(4) & =y \frac{P(3) P^{2}(0)}{P(2) P(4)}, \\
h(2)+h(1)-h(3) & =y \frac{P(2) P^{2}(0)}{P(1) P(5)}, \\
h(1)-h(2) & =\left\{\frac{P(5)}{P(3) P(4)}-y \frac{P(1)}{P(3) P(5)}+y \frac{P(3)}{P(2) P(4)}\right\} P^{2}(0),
\end{aligned}
$$


which are the coefficients of $q^{0}$ in each of (5.13)-(5.17). For the other coefficients, we substract the left-hand sides from the right-hand sides in each of (5.13)-(5.17), and see that some coefficients are zero directly, others, by the help of (b1)-(b5).

\section{REFERENCES}

[1] G. E. Andrews, The Theory of Partitions, Encyclopedia of Mathematics, Addison-Wesley, 1976. MR 58:27738

[2] G. E. Andrews and F. G. Garvan, Dyson's Crank of A Partition, Bull. Amer. Math. Soc., 18 (1988) 167-171. MR 89b:11079

[3] A. O. L. Atkin and H. P. F. Swinnerton-Dyer, Some Properties of Partitions, Proc. London Math. Soc.,(3) 4 (1954), 84-106. MR 15:685a

[4] A. O. L. Atkin and S.M. Hussain, Some Properties of Partitions (2), Trans.Amer. Math. Soc., 89 (1958), 184-200. MR 21:2635

[5] F. J. Dyson, Some Guesses in The Theory of Partitions, Eureka (Cambridge) 8 (1944) 10-15.

[6] F. J. Dyson, Mappings and Symmetries of Partitions, J. Combin. Theory Ser.A, 51 (1989) 169-180. MR 90f:05009

[7] F. G. Garvan, New Combinatorial Interpretations of Ramanujan's Partition Congruences Mod 5, 7 and 11, Trans.Amer. Math. Soc., 305 (1988), 47-77. MR 89b:11081

[8] F. G. Garvan, Generalizations of Dyson's rank and non-Rogers-Ramanujan's partitions, Manuscripta Math., 84 (1994), 343-359. MR 95e:11115

[9] R. Lewis, Relations between the rank and the crank modulo 9, J. London Math. Soc. (2) 45 (1992), 222-231. Theory 6 (1969), 56-59. MR 93e:11118

[10] A. B. Ekin, The Rank and The Crank in Theory of Partitions, Ph.D. Thesis, Sussex University, 1993.

[11] L. Winquist, An elementary proof of $p(11 m+6) \equiv 0(\bmod 11)$, J. Combin. Theory, (1969) 56-59. MR 38:4434

Ankara Üniversitesi, Fen Fakültesi, Matematik Bölümü, Tandogan, Ankara, Turkey

E-mail address: ekin@science.ankara.edu.tr 